\title{
ON SUBPARACOMPACT SPACES ${ }^{1}$
}

\author{
DENNIS K. BURKE
}

1. Introduction. This paper is concerned with establishing equivalence between several classes of topological spaces. The main result is Theorem 1.2 , for which a proof is given in $\$ 2$. Throughout this paper the set of positive integers will be denoted by $Z^{+}$.

In [2] Arhangel'skil introduces the class of $\sigma$-paracompact spaces. Following Arhangel'skir, a space $X$ is called $\sigma$-paracompact if for any open covering $\mathfrak{u}$ of $X$ there is a sequence $\left\{\mathfrak{u}_{n}\right\}_{n=1}^{\infty}$ of open coverings of $X$ such that if $x \in X$ there is $m(x) \in Z^{+}$and some set $U \in \mathcal{U}$ with $\operatorname{St}\left(x, u_{m(x)}\right) \subset U$.

The class of $\sigma$-paracompact spaces was studied recently by Čoban in [5], and the author and R. Stoltenberg in [4]. The following proposition was proved independently in both papers.

Proposition 1.1. If $X$ is a topological space with the property that every open cover of $X$ has a $\sigma$-discrete closed refinement, then $X$ is $\sigma$-paracompact.

Spaces with the property that every open cover has a $\sigma$-discrete closed refinement are called $F_{\sigma}$-screenable by McAuley in [9].

We now state a much stronger result than Proposition 1.1, which says that $\sigma$-paracompactness is actually equivalent to several conditions on a topological space and, in particular, to the $F_{\sigma}$-screenable condition.

TheOREM 1.2. For a topological space $X$ the following conditions are equivalent:

(a) $X$ is $\sigma$-paracompact.

(b) Every open cover of $X$ has a $\sigma$-discrete closed refinement.

(c) Every open cover of $X$ has a $\sigma$-locally-finite closed refinement.

(d) Every open cover of $X$ has a $\sigma$-closure-preserving closed refinement.

In view of the above theorem and because it appears that the terms $\sigma$-paracompact and $F_{\sigma}$-screenable are not very satisfactory, we make the following definition:

Definition 1.3. A topological space $X$ is called subparacompact if it satisfies any one of the conditions (a) through (d) stated in Theorem 1.2.

Received by the editors March 27, 1969.

1 This paper represents a portion of a doctoral dissertation written under the direction of Professor R. A. Stoltenberg at Washington State University. The research was partially supported by N.S.F. Grant GP-8956. 
Clearly the class of subparacompact spaces includes the class of developable ${ }^{2}$ spaces. In fact it is known that semimetric spaces [9] and semistratifiable spaces [6] satisfy (b) and hence are subparacompact.

A type of topological space which naturally possesses property (c) is a space with a $\sigma$-locally-finite closed network. ${ }^{3}$ Spaces with a $\sigma$-locally-finite network are called $\sigma$-spaces by Okuyama in [12] and [13]. Assuming regularity, Siwiec and Nagata have shown in [14] that the class of $\sigma$-spaces is equivalent to the class of spaces with a $\sigma$-discrete network and to the class of spaces with a $\sigma$-closure-preserving network.

REMARK 1.4. If $X$ is a regular space we need not require that the refinements as given in (b), (c), or (d) of Theorem 1.2 consist of closed sets.

2. Proof of Theorem 1.2. It suffices to prove $(a) \rightarrow(b)$ and $(d) \rightarrow(b)$, since it has been established by Proposition 1.1 that $(b) \rightarrow(a)$ and it is obvious that $(\mathrm{b}) \rightarrow(\mathrm{c}) \rightarrow(\mathrm{d})$.

(a) $\rightarrow$ (b). Assume $X$ satisfies condition (a) of Theorem 1.2.

Lemma 2.1. If $u$ is any open cover of $X$ there is a sequence $\left\{u_{n}\right\}_{n=1}^{\infty}$ of open covers of $X$ satisfying:

(1) $\mathfrak{u}_{1}=\mathcal{u}$ and $\mathfrak{u}_{n+1}$ refines $\mathfrak{u}_{n}$ for each $n \in Z^{+}$.

(2) Given $n \in Z^{+}, x \in X$ there is an integer $m \in Z^{+}$( $m$ depends on $n$ and $x)$ and some $U_{n} \in \mathcal{u}_{n}$ such that $\operatorname{St}\left(x, \mathfrak{u}_{m}\right) \subset U_{n}$.

Proof. By induction, for each $k \in Z^{+}$, we can find a sequence $\left\{\mathcal{u}_{n}(k)\right\}_{n=k}^{\infty}$ of open covers of $X$ such that:

(i) $\mathfrak{U}_{1}(1)=\mathcal{u}$ and $\mathfrak{u}_{k+1}(k+1)=\mathfrak{u}_{k+1}(k)$.

(ii) $\mathcal{U}_{n+1}(k)$ refines $\mathcal{U}_{n}(k)$ for each $n \geqq k$.

(iii) $\mathfrak{u}_{n}(k+1)$ refines $\mathfrak{u}_{n}(k)$ for each $n \geqq k+1$.

(iv) Given $x \in X$ there is an integer $m \in Z^{+}(m$ depends on $k$ and $x$ ) such that $\operatorname{St}\left(x, \mathfrak{u}_{m}(k)\right) \subset U_{k}$ for some $U_{k} \in \mathcal{u}_{k}(k)$.

Let $\mathfrak{U}_{k}=\mathfrak{U}_{k}(k)$; it follows that $\left\{\mathfrak{u}_{k}\right\}_{k=1}^{\infty}$ is a sequence of open covers with the desired properties.

Let $\mathcal{u}=\left\{U_{\alpha}: \alpha \in \Gamma\right\}$ be an open cover of $X$ with $\Gamma$ well-ordered. Then there is a sequence $\left\{\mathcal{u}_{n}\right\}_{n=1}^{\infty}$ of open covers of $X$ satisfying (1) and (2) of Lemma 2.1.

For each $x \in X$ let

${ }^{2}$ A sequence $\left\{\mathcal{U}_{n}\right\}_{n=1}^{\infty}$ of open covers of a space $X$ is called a development for $X$ if the collection $\left\{\operatorname{St}\left(x, \mathcal{U}_{n}\right): n \in Z^{+}\right\}$is a neighborhood base at $x$ for each $x \in X$.

${ }^{3}$ A collection $B$ of subsets of $X$ is called a network for $X$ if for any open set $O \subset X$ and $x \in O$ there is an element $B \in \mathbb{B}$ such that $x \in B \subset O$. 


$$
\Gamma_{x}=\left\{\alpha \in \Gamma: \operatorname{St}\left(x, \mathcal{u}_{n}\right) \subset U_{\alpha} \text { for some } n \in Z^{+}\right\},
$$

and define $\alpha(x)$ to be the least element of $\Gamma_{x}$.

For $\alpha \in \Gamma$ and $n \in Z^{+}$let

$$
P_{n}(\alpha)=\left\{z \in X: \operatorname{St}\left(z, \mathfrak{u}_{n}\right) \subset U_{\alpha} \text { and } \alpha=\alpha(z)\right\} .
$$

Note that $\left\{P_{n}(\alpha): \alpha \in \Gamma\right\}$ is a disjoint collection for each $n \in Z^{+}$, and the collection $\left\{P_{n}(\alpha): \alpha \in \Gamma, n \in Z^{+}\right\}$covers $X$ and refines $\mathcal{u}$.

Now if $n, m \in Z^{+}$, with $m \geqq n$, we define

$$
P_{n, m}(\alpha)=\left\{z \in P_{n}(\alpha): \operatorname{St}\left(z, \mathfrak{u}_{m}\right) \subset U_{n} \text { for some } U_{n} \in \mathcal{u}_{n}\right\} .
$$

It follows that $\bigcup_{m=n}^{\infty} P_{n, m}(\alpha)=P_{n}(\alpha)$, so the collection

$$
\mathcal{P}=\left\{P_{n, m}(\alpha): n, m \in Z^{+}, m \geqq n, \alpha \in \Gamma\right\}
$$

covers $X$ and refines $\mathcal{U}$. We will show that for a fixed $n, m \in Z^{+}, m \geqq n$, the family $\mathcal{P}_{n, m}=\left\{P_{n, m}(\alpha): \alpha \in \Gamma\right\}$ is a discrete collection of sets in $X$.

Let $P_{n, m}(\alpha)$ be a nonempty element of $P_{n, m}$ and $z$ an arbitrary element of $P_{n, m}(\alpha)$. Suppose there is some $\beta \in \Gamma, \beta \neq \alpha$, such that $\operatorname{St}\left(z, \mathcal{u}_{m}\right) \cap P_{n, m}(\beta) \neq \varnothing$. Let $y \in \operatorname{St}\left(z, \mathcal{u}_{m}\right) \cap P_{n, m}(\beta)$. Now $y \in P_{n, m}(\beta)$ implies that $\operatorname{St}\left(y, \mathfrak{u}_{m}\right) \subset U_{n}$ for some $U_{n} \in \mathfrak{u}_{n}$ and $y \in \operatorname{St}\left(z, \mathfrak{u}_{m}\right)$ implies that $z \in \operatorname{St}\left(y, \mathfrak{u}_{m}\right) \subset U_{n}$. Thus $\operatorname{St}\left(y, \mathfrak{u}_{m}\right) \subset \operatorname{St}\left(z, u_{n}\right) \subset U_{\alpha}$, so $\alpha \in \Gamma_{y}$. Hence $\beta<\alpha$ since $\beta=\alpha(y)$ and $\alpha(y)$ is the least element of $\Gamma_{y}$. Also $z \in P_{n, m}(\alpha)$ implies that $\operatorname{St}\left(z, \mathcal{U}_{m}\right) \subset U_{n}^{\prime}$ for some $U_{n}^{\prime} \in \mathfrak{U}_{n}$. But $y \in \operatorname{St}\left(z, u_{m}\right) \subset U_{n}^{\prime}$ implies that $\operatorname{St}\left(z, u_{m}\right) \subset \operatorname{St}\left(y, \mathfrak{u}_{n}\right) \subset U_{\beta}$, so $\beta \in \Gamma_{z}$. Hence $\alpha(z)=\alpha<\beta$ and we have a contradiction.

Since $z$ was an arbitrary element of $P_{n, m}(\alpha)$ we have shown that $\operatorname{St}\left(P_{n, m}(\alpha), u_{m}\right) \cap P_{n, m}(\beta)=\varnothing$ whenever $\beta \neq \alpha$.

To complete the proof that $\mathcal{P}_{n, m}$ is discrete we show for each $x \in X$ that there is a neighborhood of $x$ which intersects at most one element of $P_{n, m}$. Let $P_{n, m}=U\left\{P_{n, m}(\alpha): \alpha \in \Gamma\right\}$. If $x \in X-\bar{P}_{n, m}$ we are through, so suppose $x \in \bar{P}_{n, m}$. Now

$$
\begin{aligned}
P_{n, m} & \subset X-U\left\{U \in \mathcal{u}_{m}: U \cap P_{n, m}=\varnothing\right\} \\
& \subset U\left\{\operatorname{St}\left(P_{n, m}(\alpha), u_{m}\right): \alpha \in \Gamma\right\}=\operatorname{St}\left(P_{n, m}, u_{m}\right)
\end{aligned}
$$

and $X-\cup\left\{U \in \mathfrak{u}_{m}: U \cap P_{n, m}=\varnothing\right\}$ is a closed set. Thus $\bar{P}_{n, m}$ $C \operatorname{St}\left(P_{n, m}, \mathfrak{u}_{m}\right)$. So $x \in \operatorname{St}\left(P_{n, m}, \mathfrak{u}_{m}\right)$ and there must be some $\beta \in \Gamma$ such that $x \in \operatorname{St}\left(P_{n, m}(\beta), \mathcal{U}_{m}\right)$. We have shown above that $\operatorname{St}\left(P_{n, m}(\beta), \mathfrak{u}_{m}\right)$ does not intersect any $P_{n, m}(\alpha)$ for $\alpha \neq \beta$. Hence $\operatorname{St}\left(P_{n, m}(\beta), \mathfrak{u}_{m}\right)$ is a neighborhood of $x$ which intersects only one element of $\Theta_{n, m}$. It follows that $\mathcal{P}=\bigcup_{n=1}^{\infty} \bigcup_{m=n}^{\infty} \mathcal{P}_{n, m}$ is a $\sigma$-discrete refinement of $\mathcal{u}$.

To complete the proof that $(\mathrm{a}) \rightarrow$ (b) note that if $P_{n, m}(\alpha) \in \mathcal{P}_{n, m}$, 
then $\mathrm{Cl}\left(P_{n, m}(\alpha)\right) \subset \bar{P}_{n, m} \subset \bigcup\left\{\operatorname{St}\left(P_{n, m}(\beta), \mathfrak{u}_{m}\right): \beta \in \Gamma\right\}$ and $\mathrm{Cl}\left(P_{n, m}(\alpha)\right)$ $\cap \operatorname{St}\left(P_{n, m}(\beta), \mathfrak{u}_{m}\right)=\varnothing$ for $\beta \neq \alpha$. Thus $\mathrm{Cl}\left(P_{n, m}(\alpha)\right) \subset \operatorname{St}\left(P_{n, m}(\alpha), \mathfrak{u}_{m}\right)$ $\subset \operatorname{St}\left(P_{n, m}(\alpha), \mathfrak{u}_{n}\right) \subset U_{\alpha}$. We have shown that

$$
\mathcal{P}^{\prime}=\left\{\mathrm{Cl}\left(P_{n, m}(\alpha)\right): P_{n, m}(\alpha) \in \mathcal{Q}\right\}
$$

is a closed refinement of $\mathcal{u}$. Since $\beta$ is $\sigma$-discrete, $\beta^{\prime}$ is a $\sigma$-discrete closed refinement.

(d) $\rightarrow$ (b). Assume every open cover of $X$ has a $\sigma$-closure-preserving closed refinement.

Lemma 2.2. For each $n \in Z^{+}$, let $\mathfrak{u}(n)=\left\{U_{\alpha}(n): \alpha \in \Gamma\right\}$ be an open cover of $X$ such that $U_{\alpha}(n+1) \subset U_{\alpha}(n)$ for all $\alpha \in \Gamma$. Then there is a sequence $\{P(n)\}_{n=1}^{\infty}$ of closed coverings of $X$ such that, for each $n \in Z^{+}$, $P(n)=\bigcup_{m=1}^{\infty} P_{m}(n)$ and the following conditions are satisfied:

(1) $\mathcal{P}_{m}(n)=\left\{P_{\alpha, m}(n): \alpha \in \Gamma\right\}$ and is closure-preserving for each $m \in Z^{+}$.

(2) $P_{\alpha, m}(n) \subset U_{\alpha}(n)$ for each $\alpha \in \Gamma, m \in Z^{+}$.

(3) $P_{\alpha, m}(n) \subset P_{\alpha, m+1}(n)$ for each $\alpha \in \Gamma, m \in Z^{+}$.

(4) $P_{\alpha, m}(n+1) \subset P_{\alpha, m}(n)$ for each $\alpha \in \Gamma, m \in Z^{+}$.

Proof. Each $u(n)$ has a closed refinement $B(n)=\cup_{m=1}^{\infty} B_{m}(n)$ where each $\beta_{m}(n)$ is closure-preserving and $\beta_{m}(n) \subset \Theta_{m+1}(n)$. Let $m, n \in Z^{+}$and $\alpha \in \Gamma$. If $m<n$ let $P_{\alpha, m}(n)=\varnothing$. If $m \geqq n$ define

$$
P_{\alpha, m}(n)=U\left\{B \in B_{m}(k): B \subset U_{\alpha}(n), k \in Z^{+}, n \leqq k \leqq m\right\} .
$$

Let $P_{m}(n)=\left\{P_{\alpha, m}(n): \alpha \in \Gamma\right\}$ and $P(n)=\bigcup_{m=1}^{\infty} \odot_{m}(n)$. It is easily verified that $\{\odot(n)\}_{n=1}^{\infty}$ is the required sequence of closed covers.

Let $\mathcal{u}=\left\{U_{\alpha}: \alpha \in \Gamma\right\}$ be an open cover of $X$ with $\Gamma$ well ordered. We will construct a $\sigma$-discrete closed refinement of $\mathcal{u}$. For notational purposes let $U_{\alpha}(1, n)=U_{\alpha}$ and $\mathcal{u}(1, n)=\left\{U_{\alpha}(1, n): \alpha \in \Gamma\right\}$ for each $n \in Z^{+}$. There is a sequence $\{\odot(1, n)\}_{n=1}^{\infty}$ of closed covers of $X$ such that $\mathcal{P}(1, n)=\mathrm{U}_{m=1}^{\infty} \mathcal{P}_{m}(1, n)$ and conditions (1) through (4) of Lemma 2.2 are satisfied, with the obvious notational changes. It follows by induction that, for each $k \in Z^{+}$, we can find a sequence $\{u(k, n)\}_{n=1}^{\infty}$ of open covers and a sequence $\{\mathcal{P}(k, n)\}_{n=1}^{\infty}$ of closed covers of $X$ such that $\mathcal{U}(k, n)=\left\{U_{\alpha}(k, n): \alpha \in \Gamma\right\}, \odot(k, n)=\bigcup_{m=1}^{\infty} \odot_{m}(k, n)$, and the following is true for each $m, n \in Z^{+}$:

(1) $P_{m}(k, n)=\left\{P_{\alpha, m}(k, n): \alpha \in \Gamma\right\}$ is a closure-preserving collection.

(2) $P_{\alpha, m}(k, n) \subset U_{\alpha}(k, n)$,

(3) $P_{\alpha, m}(k, n) \subset P_{\alpha, m+1}(k, n)$,

(4) $P_{\alpha, m}(k, n+1) \subset P_{\alpha, m}(k, n)$,

(5) $U_{\alpha}(k+1, n)=U_{\alpha}-U_{\beta<\alpha} P_{\beta, n}(k, 1)$.

Notice that for $x \in X$, if $\alpha(x)$ is the first element of $\Gamma$ such that 
$x \in U_{\alpha(x)}$, then $x \in U_{\alpha(x)}(k, n)$ for any $k, n \in Z^{+}$. Hence $\mathfrak{u}(k, n)$ is indeed an open cover of $X$.

For $k, m, n \in Z^{+}$and $\alpha \in \Gamma$ define

$$
L_{\alpha}(k, m, n)=P_{\alpha, n}(k, 1) \cap P_{\alpha, m}(k+1, n) .
$$

Then $\mathcal{L}(k, m, n)=\left\{L_{\alpha}(k, m, n): \alpha \in \Gamma\right\}$ is a closure-preserving collection of closed sets. Let $\gamma, \alpha \in \Gamma$ with $\gamma \neq \alpha$; say $\gamma<\alpha$. Then

$$
\begin{aligned}
& L_{\alpha}(k, m, n) \subset P_{\alpha, m}(k+1, n) \subset U_{\alpha}(k+1, n) \\
& =U_{\alpha}-\bigcup_{\beta<\alpha} P_{\beta, n}(k, 1) \subset U_{\alpha}-P_{\gamma, n}(k, 1) \subset U_{\alpha}-L_{\gamma}(k, m, n) .
\end{aligned}
$$

Hence $L_{\gamma}(k, m, n) \cap L_{\alpha}(k, m, n)=\varnothing$ when $\gamma \neq \alpha$. Thus $\mathfrak{L}(k, m, n)$ is a discrete collection of closed sets for each $k, m, n \in Z^{+}$. Clearly $L_{\alpha}(k, m, n) \subset U_{\alpha}$, so we are through if we show $\mathscr{L}=\bigcup_{k=1}^{\infty} \bigcup_{m=1}^{\infty} \bigcup_{n=1}^{\infty}$ $\mathfrak{L}(k, m, n)$ covers $X$.

Let $x \in X$. Since $\Gamma$ is well ordered, there is a $\beta \in \Gamma$, and $k, m, n \in Z^{+}$, such that $x \in P_{\beta, n}(k, m)$ but $x \in P_{\alpha, n^{\prime}}\left(k^{\prime}, m^{\prime}\right)$ if $\alpha<\beta$ and $k^{\prime}, m^{\prime}$, $n^{\prime} \in Z^{+}$. We show that $x \in L_{\beta}(k, m, n)$. First notice that, if $\alpha>\beta$, $P_{\alpha, m}(k+1, n) \subset U_{\alpha}(k+1, n) \subset U_{\alpha}-P_{\beta, n}(k, 1)$ and so $P_{\beta, n}(k, 1)$ $\cap P_{\alpha, m}(k+1, n)=\varnothing$. Thus

$$
\begin{aligned}
x & \in P_{\beta, n}(k, m)-\bigcup_{\alpha<\beta} P_{\alpha, m}(k+1, n) \\
& \subset P_{\beta, n}(k, 1)-\bigcup_{\alpha<\beta} P_{\alpha, m}(k+1, n) \\
& =P_{\beta, n}(k, 1)-\bigcup_{\alpha \neq \beta} P_{\alpha, m}(k+1, n) \\
& \subset P_{\beta, n}(k, 1) \cap P_{\beta, m}(k+1, n)=L_{\beta}(k, m, n),
\end{aligned}
$$

and that completes the proof.

3. Properties of subparacompact spaces. This section is devoted to showing a few properties of subparacompact spaces. Most of the results follow in a straightforward manner by the use of Theorem 1.2. Theorems 3.1 and 3.2 answer questions asked by Arhangel'skil in [2].

TheOREM 3.1. If $f: X \rightarrow Y$ is a closed map from a subparacompact space $X$ onto $Y$ then $Y$ is subparacompact.

Proof. If $u$ is any open cover of $Y, f^{-1}(\mathcal{u})=\left\{f^{-1}(U): U \in \mathcal{u}\right\}$ is an open cover of $X$. Since $X$ is subparacompact $f^{-1}(\mathcal{U})$ has a closed refinement $P=\bigcup_{n=1}^{\infty} \rho_{n}$ where $P_{n}$ is a closure-preserving collection of closed sets for each $n \in Z^{+}$. Since $f$ is a closed map it follows that $f\left(\odot_{n}\right)=\left\{f(P): P \in \odot_{n}\right\}$ is a closure-preserving collection of closed sets 
in $Y$. Thus $f(\mathcal{P})$ is a $\sigma$-closure-preserving refinement of $\mathcal{u}$ and $Y$ is subparacompact.

Theorem 3.2. If $f: X \rightarrow Y$ is a perfect map from a regular space $X$ onto a subparacompact space $Y$ then $X$ is subparacompact.

Proof. Suppose $\mathcal{U}$ is any open cover of $X$. For each $y \in Y$ we can find a finite subcollection $\mathfrak{u}(y) \subset \mathfrak{U}$ such that $f^{-1}(y) \subset U(y)=$ $U\{U: U \in \mathcal{u}(y)\}$. Let $G(y)=Y-f(X-U(y))$; then $\mathcal{G}=\{G(y): y \in Y\}$ is an open cover of $Y$. Consequently $\mathcal{G}$ has a $\sigma$-discrete refinement $\mathcal{P}=\cup_{n=1}^{\infty} \mathcal{P}_{n}$ where each $\mathcal{P}_{n}$ is a discrete collection. It follows that $f^{-1}(P)=\left\{f^{-1}(P): P \in \mathcal{P}\right\}$ is a $\sigma$-discrete refinement of $\{U(y): y \in Y\}$. Given $P \in \mathcal{P}$ let $y(P)$ be a fixed element of $Y$ such that $f^{-1}(P) \subset U(y(P))$. For $n \in Z^{+}$let $\mathbb{B}_{n}=\left\{f^{-1}(P) \cap U: P \in \mathcal{P}_{n}, U \in \mathcal{U}(y(P))\right\}$. Since $f^{-1}\left(P_{n}\right)$ is a discrete collection in $X$ each $x \in X$ has a neighborhood $N_{x}$ which intersects at most one element of $f^{-1}\left(\odot_{n}\right)$. Since each element of $f^{-1}\left(\mathcal{P}_{n}\right)$ intersects only finitely many elements of $\Theta_{n}$ and each element of $\beta_{n}$ is contained in some element of $P_{n}$ it follows that $N_{x}$ will intersect only finitely many elements of $\Theta_{n}$. So $\Theta_{n}$ is locally-finite and $B=\bigcup_{n=1}^{\infty} B_{n}$ is a $\sigma$-locally-finite refinement of $u$. Since $X$ is regular it follows that every open cover of $X$ has a $\sigma$-locally-finite closed refinement. Hence $X$ is subparacompact.

A completely regular space $X$ is called a $p$-space $[1]$ if in the StoneČech compactification $\beta(X)$ there is a sequence $\left\{\gamma_{n}\right\}_{n=1}^{\infty}$ of open covers of $X$ such that $\bigcap_{n=1}^{\infty} \operatorname{St}\left(x, \gamma_{n}\right) \subset X$ for each $x \in X$. The sequence $\left\{\gamma_{n}\right\}_{n=1}^{\infty}$ is called a pluming for $X$ in $\beta(X) . X$ is a strict p-space if there is a pluming $\left\{\gamma_{n}\right\}_{n=1}^{\infty}$ with the additional property: $\bigcap_{n=1}^{\infty} \operatorname{Cl}\left(\operatorname{St}\left(x, \gamma_{n}\right)\right)$ $=\bigcap_{n=1}^{\infty} \operatorname{St}\left(x, \gamma_{n}\right)$ for each $x \in X$.

By Theorem 3.2 a regular space which is the perfect preimage of a developable space is subparacompact; if this preimage is completely regular it can be shown to be a $p$-space. Since Arhangel'skir [2] announced that any $\sigma$-paracompact $p$-space can be mapped perfectly onto a developable space, we have the following corollary to Theorem 3.2.

Corollary 3.3. A completely regular space $X$ can be mapped onto a developable space by a perfect map if and only if $X$ is a subparacompact p-space.

In [2] Arhangel'skil asks whether every strict $p$-space can be mapped perfectly onto a developable space. To answer this question negatively it suffices to find an example of a strict $p$-space which is not subparacompact. Such an example will be given in the last section of this paper. 
TheOREM 3.4. A collectionwise normal subparacompact space is paracompact.

Theorem 3.4 is a restatement of a result by McAuley [9] that a collectionwise normal $F_{\sigma}$-screenable space is paracompact.

Since $\sigma$-discrete families in a countably compact space must be countable the following is obvious:

TheOREM 3.5. A countably compact, subparacompact space $X$ is compact.

Theorem 3.6. A subparacompact locally-developable space $X$ is developable.

Proof. Suppose each point $x \in X$ has an open neighborhood $N_{x}$ with a development $\left\{g_{n}(x)\right\}_{n=1}^{\infty}$. Let $\odot=\cup_{n=1}^{\infty} \otimes_{n}$ be a $\sigma$-discrete closed refinement of $\left\{N_{x}: x \in X\right\}$ where each $P_{n}$ is a discrete collection.

Now fix an arbitrary positive integer $n$. Given $P \in \mathcal{P}_{n}$ let $x(P)$ be a fixed element of $X$ such that $P \subset N_{x(P)}$ and let

$$
U(P)=X-U\left\{P^{\prime} \in \mathcal{P}_{n}: P^{\prime} \neq P\right\} .
$$

For $m \in Z^{+}$let

$$
\mathfrak{u}_{n, m}(P)=\left\{U(P) \cap G: G \in \mathcal{S}_{m}(x(P))\right\} .
$$

Finally we define

$$
\mathcal{u}_{n, m}=\left\{U: U \in \mathcal{u}_{n, m}(P), P \in \mathcal{P}_{n}\right\} \cup\left\{Q_{n}\right\}
$$

where $Q_{n}=X-\cup\left\{P: P \in \odot_{n}\right\}$. Then $\mathcal{U}_{n, m}$ is an open cover of $X$ for each $n, m \in Z^{+}$and we show that $\left\{u_{n, m}: n, m \in Z^{+}\right\}$is a development for $X$.

If $z \in X$ there is an integer $n \in Z^{+}$with some $P \in \odot_{n}$ such that $z \in P$. Consequently if $O$ is any open set containing $z$ there is some $m \in Z^{+}$ such that $\operatorname{St}\left(z, \mathrm{G}_{m}(x(P))\right) \subset O \cap N_{x(P)}$. By construction $z$ is not contained in any element of $\mathcal{u}_{n, m}\left(P^{\prime}\right)$ for any $P^{\prime} \in \mathcal{P}_{n}$ such that $P^{\prime} \neq P$. Thus $\operatorname{St}\left(z, \mathfrak{u}_{n, m}\right)=\operatorname{St}\left(z, \mathfrak{u}_{n, m}(P)\right) \subset \operatorname{St}\left(z, \mathcal{g}_{m}(x(P))\right) \subset O \cap N_{x(P)} \subset O$. Hence $\operatorname{St}\left(z, \mathfrak{u}_{n, m}\right) \subset O$ and the theorem is proved.

The proof of the following theorem is straightforward and is left to the reader.

THeOREM 3.7. If $X$ is a countable union of closed subparacompact subspaces then $X$ is subparacompact.

\section{Examples.}

EXAMPLE 4.1. A strict $p$-space which is not subparacompact and hence cannot be mapped perfectly onto a developable space. 
Let $\omega_{1}$ (resp. $\omega_{2}$ ) be the first ordinal of cardinality $\boldsymbol{\aleph}_{1}$ (resp. $\boldsymbol{\aleph}_{2}$ ). If $X$ is the set of points in the cartesian product of $\left[0, \omega_{2}\right)$ with itself and $\alpha \in\left(0, \omega_{2}\right)$ we define:

$L_{1, \alpha}=\left\{(\alpha, \beta) \in X: \beta \in\left[0, \omega_{2}\right)\right\}$ and $L_{2, \alpha}=\left\{(\beta, \alpha) \in X: \beta \in\left[0, \omega_{2}\right)\right\}$. For convenience let $L_{1,0}=L_{2,0}=\{(0,0)\}$.

Define an open base for a topology on $X$ as follows: For each $(\alpha, \beta) \in X$ where $\alpha \neq 0$ and $\beta \neq 0$ the singleton set $\{(\alpha, \beta)\}$ is in the base. For $i=1,2$ and $\alpha \in\left[0, \omega_{2}\right)$ the base includes all subsets of $L_{i, \alpha}$ which have a finite complement relative to $L_{i, \alpha}$. It is easily verified that this collection does form an open base for a topology on $X$.

Notice that with the above described topology $X$ is a $T_{2}$ locally compact space. Hence $X$ is open in $\beta(X)$. That $X$ is a strict $p$-space follows from the definition if, for each $n \in Z^{+}$, we let

$$
\mathcal{S}=\gamma_{n}=\left\{L_{i, \alpha}: \alpha \in\left[0, \omega_{2}\right), \quad i=1,2\right\} .
$$

Then $\gamma_{n}$ is a cover of $X$ open in $\beta(X)$ and $\operatorname{St}\left(x, \gamma_{n}\right)$ is an open compact set for each $x \in X$.

The following lemma will be needed to show that $X$ is not subparacompact.

Lemma. Suppose $A$ and $B$ are subsets of $X$ with the property: $A \cap L_{1, \alpha}$ and $B \cap L_{2, \alpha}$ are countable for each $\alpha \in\left[0, \omega_{2}\right)$. Then $A$ and $B$ do not cover $X$.

Proof. Suppose $X=A \cup B$. Let

$$
\beta_{0}=\sup \left\{\beta:(\alpha, \beta) \in A \cap L_{1, \alpha}, \alpha \in\left[0, \omega_{1}\right)\right\} .
$$

Then $\beta_{0}<\omega_{2}$ and it follows that $\left[0, \omega_{1}\right) \times\left(\beta_{0}, \omega_{2}\right)$ is a nonempty subset of $X$ disjoint from $A$. Thus $\left[0, \omega_{1}\right) \times\left(\beta_{0}, \omega_{2}\right) \subset B$. Let $\alpha_{0} \in\left(\beta_{0}, \omega_{2}\right)$. Then $\left[0, \omega_{1}\right) \times\left\{\alpha_{0}\right\} \subset B \cap L_{2, \alpha_{0}}$ which is in contradiction with the original condition placed on $B$.

We show that $X$ is not subparacompact by showing that $G$ cannot have a $\sigma$-discrete refinement.

Assume $\mathcal{P}=\cup_{n=1}^{\infty} \rho_{n}$ is a refinement of $\mathcal{G}$ where each $\rho_{n}$ is a discrete collection. Let $\mathcal{G}^{\prime}=\left\{L_{1, \alpha}: \alpha \in\left[0, \omega_{2}\right)\right\}$ and $\mathcal{G}^{\prime \prime}=\left\{L_{2, \alpha}: \alpha \in\left[0, \omega_{2}\right)\right\}$; then $\mathcal{G}^{\prime}$ and $\mathcal{G}^{\prime \prime}$ are subcollections of $\mathcal{G}$. Let $\mathcal{P}_{n}^{\prime}$ and $\mathcal{P}_{n}^{\prime \prime}$ be all elements of $\mathcal{P}_{n}$ which are contained in elements of $\mathcal{G}^{\prime}$ and $\mathcal{G}^{\prime \prime}$ respectively. Note that every element of $\mathcal{P}_{n}$ will be in $\mathcal{P}_{n}^{\prime}$ or $\mathcal{P}_{n}^{\prime \prime}$. Let $P_{n}^{\prime}$ and $P_{n}^{\prime \prime}$ be the union of all the elements in $\mathcal{P}_{n}^{\prime}$ and $\mathcal{P}_{n}^{\prime \prime}$ respectively. If $\alpha \in\left[0, \omega_{2}\right)$ there is a neighborhood of $(\alpha, 0)$ which intersects at most one element of $P_{n}^{\prime \prime}$. Hence $L_{1, \alpha}$ intersects only finitely many elements of $\rho_{n}^{\prime \prime}$ and it follows that $P_{n}^{\prime \prime} \cap L_{1, \alpha}$ is finite. Similarly $P_{n}^{\prime} \cap L_{2, \alpha}$ is finite. Let $A=\bigcup_{n=1}^{\infty} P_{n}^{\prime \prime}$ and $B=\bigcup_{n=1}^{\infty} P_{n}^{\prime}$. Then $A$ and $B$ satisfy the conditions 
in the lemma and consequently cannot cover $X$. Thus $\beta$ does not cover $X$.

EXAMPle 4.2. A pointwise paracompact space which is not subparacompact.

It is easily verified that the space of Example 4.1 is pointwise paracompact.

EXAMPLE 4.3. A pseudocompact subparacompact space which is not countably compact.

Let $X$ be the set of points in the unit interval $[0,1]$ with nonzero points having their usual neighborhoods. Let the neighborhood system at zero include the usual neighborhoods minus the points in the sequence $\{1 / n\}_{n=1}^{\infty}$.

EXAMPLE 4.4. A normal, countably paracompact, subparacompact space which is not paracompact.

Let $X$ be the space of Example $H$ described by Bing in [3]. This space is perfectly normal and hence countably paracompact [7]. $X$ is subparacompact since it can be shown to possess a $\sigma$-discrete network; however it is not paracompact since it is not collectionwise normal.

\section{REFERENCES}

1. A. V. Arhangel'skiir, On a class of spaces containing all metric and all locally bicompact spaces, Dokl. Akad. Nauk SSSR 151 (1963), 751-754=Soviet Math. Dokl. 4 (1963), 1051-1055.

2. - Mappings and spaces, Russian Math. Surveys 21 (1966), 115-162. 186.

3. R. H. Bing, Metrization of topological spaces, Canad. J. Math. 3 (1951), 175-

4. D. K. Burke and R. A. Stoltenberg, A note on p-spaces and Moore spaces, Pacific J. Math (to appear).

5. M. M. Coban, On $\sigma$-paracompact spaces, Vestnik Moskov. Univ. Ser. I Mat. Meh. (1969), 20-27.

6. G. Creede, Semi-stratifiable spaces, Topology Conference, Arizona State University, Tempe, Ariz., 1967, pp. 318-323.

7. C. H. Dowker, On countably paracompact spaces, Canad. J. Math. 3 (1951), 219-224.

8. J. Dugundji, Topology, Allyn and Bacon, Boston, 1966.

9. L. F. McAuley, A note on complete collectionwise normality and paracompactness, Proc. Amer. Math. Soc. 9 (1958), 796-799.

10. E. Michael, Another note on paracompact spaces, Proc. Amer. Math. Soc. 8 (1957), 822-828.

11. A. Okuyama, Some generalizations of metric spaces, their metrization theorems and product spaces, Sci. Rep. Tokyo Kyoiku Daigaku Sect. A 9 (1967), 236-254.

12. - $\sigma$-spaces and closed mappings. I, Proc. Japan Acad. 44 (1968), 472-477.

13. —, $\sigma$-spaces and closed mappings. II, Proc. Japan Acad. 44 (1968), 478481.

14. F. Siwiec and J. Nagata, $A$ note on nets and metrization, Proc. Japan Acad. 44 (1968), 623-627.

WASHiNGTON State University 